\title{
Concept and implementation of an agent-based control architecture for a cyber-physical assembly system
}

\author{
Prof. Dr.-Ing. Rainer Müller ${ }^{1}$, Dipl.-Wirt.-Ing. (FH) Matthias Vette M.Eng ${ }^{1}$, Dipl.-Ing. Dipl.-Wirt.Ing. Leenhard Hörauf ${ }^{1}$, \\ Dipl.-Ing. Christoph Speicher ${ }^{1,}$ and Kruttika Jatti M.Sc ${ }^{1}$ \\ ${ }^{1}$ Centre for Mechatronics and Automatin g $\mathrm{GmbH}$ (ZeMA) \\ Group of Assembly Systems and Automation Technology \\ Gewerbepark Eschberger Weg 46, Geb. 9, 66121 Saarbrücken, Germany
}

\begin{abstract}
Today's production companies are challenged with: the assembly of different product variants with lot size of one on a single assembly line and the increase of product variants. The implementation of reconfigurable assembly systems with a modular design is a solution, which allows a flexible adaptation to different product variants. However, handling different assembly modules via specific communication protocols is limited by the current centralized control concepts. Therefore, this paper presents a concept for an agent-based control architecture. This concept uses coherent information starting from the planning process of the assembly system until the system is put into operation allowing a decentralized control. The decentralized agents in each station act as mediators between the modules and the assembly memory database enabling the product autonomous control throughout the assembly system. Moreover, the agent manages the data transfer for the modules with different specific syntaxes via respective communication protocols since a standardized communication protocol does not exist in industry. The concept is verified in a test assembly line for the production of small electronic components.
\end{abstract}

\section{Introduction}

Nowadays production companies are facing a growing number of product variants, which need to be assembled while batch sizes and product life cycles are continuously decreasing [1]. Multi-variant lines enable the assembly of several product variants on the same assembly line. These cyber-physical systems consist of several modules known as intelligent embedded systems, which sense their environment by sensors and interact with the environment via actuators [2]. The modules of these systems can be reconfigured depending on the products that need to be assembled. However, this flexibility results in an increasing complexity in the planning process, especially in developing the control of the system. This is due to the fact that standardized protocols for the data exchange between each other, the modules and between higher automation layers do not exist.

Due to the growing complexity in handling communication protocols between different modules, this paper presents and implements the concept of an agent-based control system for a cyber-physical assembly system into a demonstrator assembly line. The agent acts as a flexible data processing application with data input and output interfaces, so the communication between different modules can be handled. To allow reconfiguration and adaption of the modules within the stations, a flexible approach has to be chosen, so that the agent's functionality can be adapted easily in the control layer. Therefore a standardized semantic has to be implemented for the description of each assembly operation. The approach allows triggering different modules based on the product requirements, which are received from the database based on the identified product.

\section{State of the art: From automation pyramid to corporate control networks}

The "automation pyramid" is a structured hierarchical system in which each automation level helps to perform a specific automation function. The pyramid has five levels where each level exchanges information only to its adjacent layers. Due to remarkable innovations in information technology, the traditional automation pyramid's hierarchy architecture is dissolving at an increased rate urging the need to shift from centralized to decentralized architecture. The idea of decentralized automation faces the challenges encountered by production industries, such as rapid production changing conditions, increased product variants, reduced batch sizes, decreased product life cycles, etc.. Thus, the use of 
decentralized automation systems helps in achieving increased flexibility, higher efficiency and overall adaptability in automation systems [3].

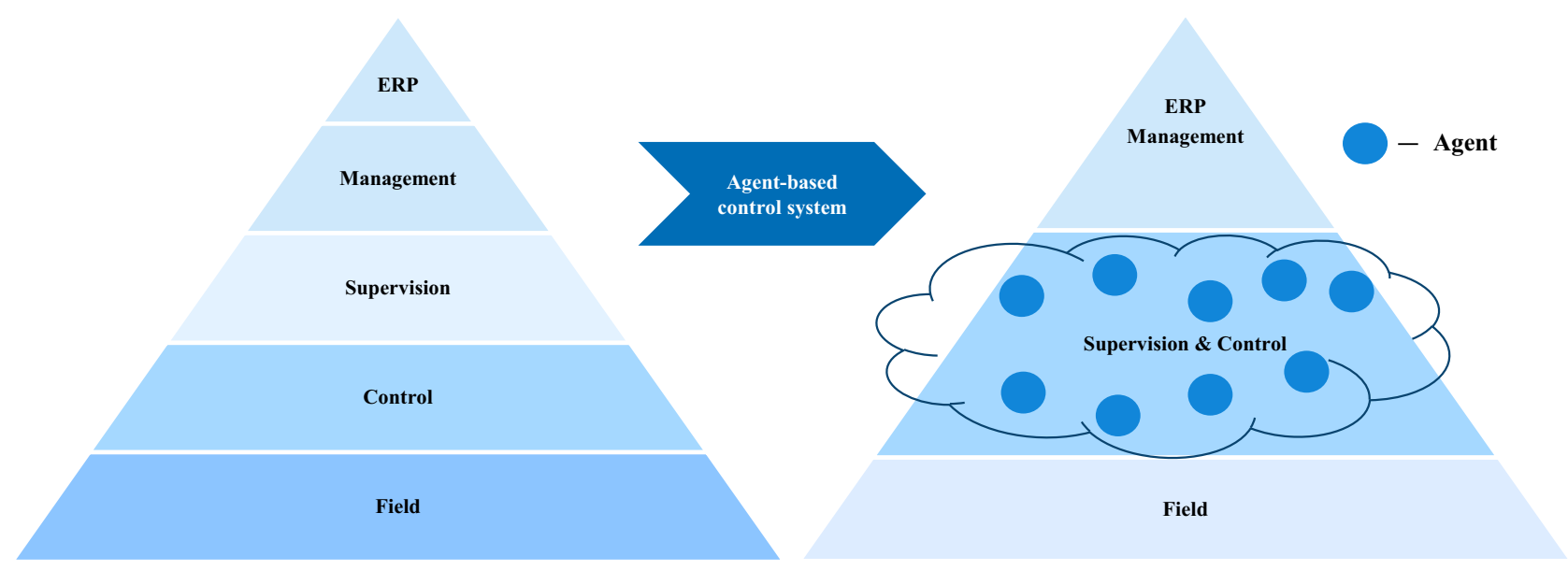

Figure 1: Transition from the classical automation pyramid to an agent-based control architecture

Cyber-physical production systems can be abstracted as the decomposition of the automation pyramid to decentralized systems, making information available everywhere and at any time [4]. To realize the system of corporate control networks agents and reconfigurable assembly systems (RAS) are used. RAS have the capacity to enable the fast exchange and adjustment of different product variants of the modules in the assembly line. The concept also enables a fast exchange of the modules of the assembly line itself without undergoing complex reconfigurational steps [5].Currently agent based control systems are considered as an important approach in developing agile distributed production systems [6]. A software agent is a "computational system that is situated in a dynamic environment and is capable of exhibiting autonomous and intelligent behavior" [7]. Although the term agent and RAS go back to at least a quarter of a century ago they have barely made a significant breakthrough in the last decade. There are still many hurdles to overcome in order to develop a fully decentralized system, and there are just a handful of real scenarios where such a system has been implemented in the production industry [8].

\section{Concept for an agent-based control architecture}

The RAS is developed by assembly planners who are analyzing the structure of the product, the assembly operations and the required assembly modules [9]. Furthermore, the assembly operations and modules are mapped into the stations of the assembly line in a virtual planning environment. This guarantees that the product variant can be produced on the assembly line with its current configuration, before the product enters into the line [10]. The data generated during this stage of the assembly planning is further used to control the navigation process of the product through the assembly system with the help of the derived assembly memory as shown in Fig. 2. In the assembly memory, the operations of each product variant are described by a basic task, a specific task, parameters, and the affiliated detailed information of the assembly modules. The information stored in the assembly memory is semantically the same as the information used in the assembly planning. This approach simplifies and improves the integration of new product variants as well as the adaption of existing variants.

The objective of the agent-based control architecture is to switch from the traditional centralized hierarchical control architecture to the distributed control architecture by using a flexible data processing application. This application as an agent is able to read, process, write and exchange data via interfaces within an assembly system. An agent is set up at each station of the assembly line and has access to a database where the assembly memory is stored. The different station modules, which offer a specific ability, are connected to the agent as shown in Fig. 2. Via the different interfaces, the modules and agent are able to exchange the information from the assembly memory. The agent acts as a mediator or coordinator to the various cyber-physical modules. The main task of the agent is to make decisions based on the received information from the database, to format it into appropriate syntax that can be processed by the relevant module and return a result. Furthermore, the module processes the received parameters and performs the requested assembly operations. This results in an event based module triggering by the information derived from the assembly memory instead of a fixed sequence chain. This has the advantage that the modules perform their operation based on the received parameters. Moreover, new modules can be easily attached to the agent by configuring the required interface and formatter inside the agent.

When a product enters a station, the transport module identifies the product and sends the identifier to the agent. Based on this identifier, the agent requests information 
about the operation needed to be performed on that particular product from the database. Based on the returned result, a decision is made whether the product is requesting a service from one of the modules within the station. If no services are to be performed at the station, the product is sent to the next station containing the relevant modules. Else, if the station possesses the relevant module, the agent will process and format the received information. This includes the tasks, parameters, and specific syntax that are read from the module via its respective communication protocol. When the module successfully completes the assembly operation, the agent is informed and updates the assembly memory of the product variant so that the next operation can be started.

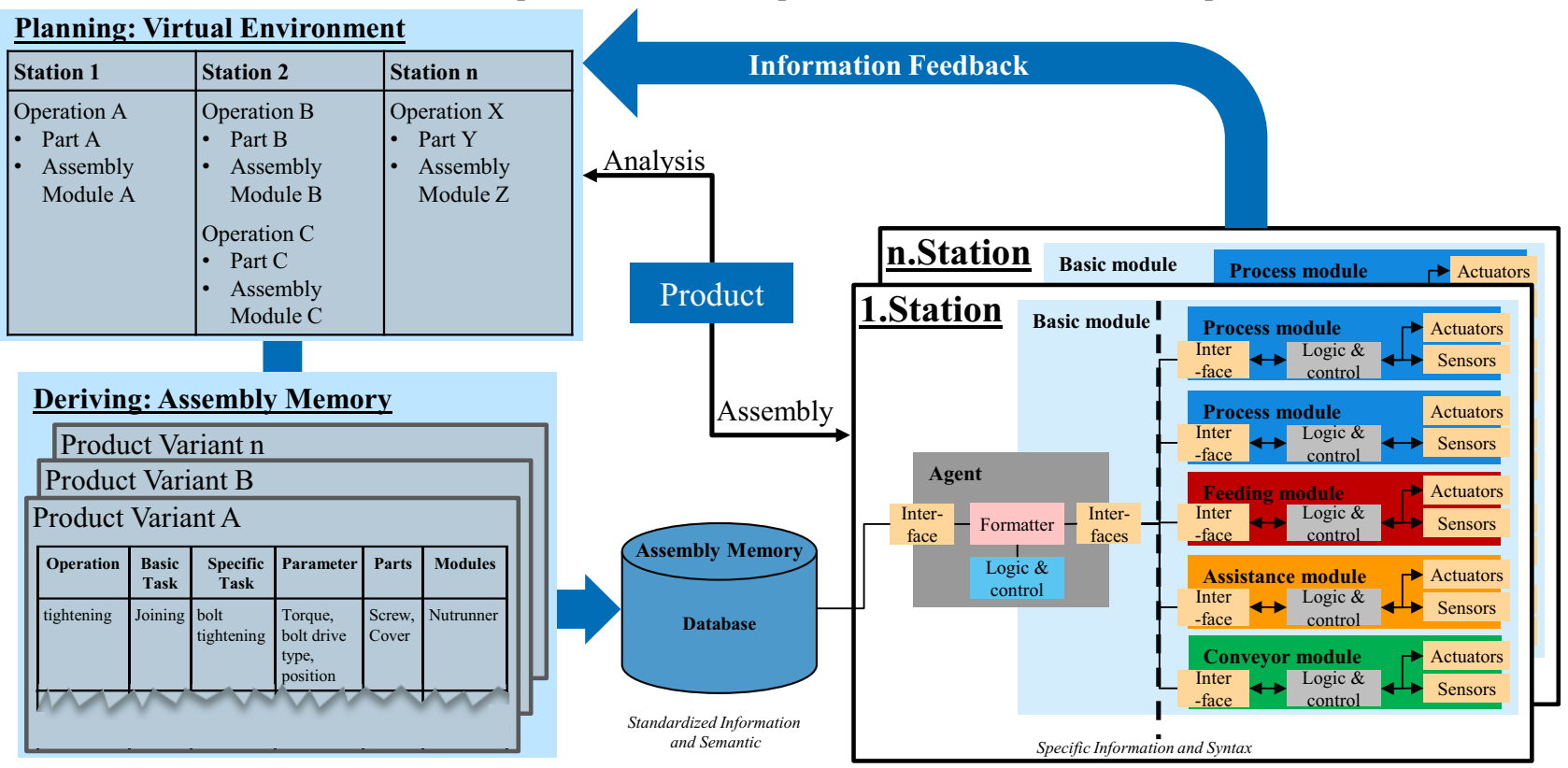

Figure 2: Concept for consistent information usage and agent-based control

\section{Implementation of the control architecture in a cyber-physical research environment}

For the validation of this control concept, the ZeMA research center implemented a cyber-physical demonstrator line. In this assembly line, an electronic micro control unit with a wide product range and various components is assembled as the product, illustrated in Fig. 3. The assembly of the product involves several processes, starting with the placement of the top casing onto the work piece carrier, then followed by assembling the inner electronic parts and ending with the bottom casing assembly. At the end, the product is inspected for functionality and, if necessary, reworked. The assembly operations are scheduled at various stations. The variation of parts is achieved by assembling different casing shapes and different circuit board designs. Variations of process task parameters are also created. For example, a bolttightening task is done using different drive types, torques, and positions or by using different types of joining processes altogether.

The demonstrator assembly line consists of four independent workstations and three transfer stations possessing a specific set of modules. In the first three stations, the products are assembled whereas in the fourth station the reworking takes place. The transportation of the product between stations is done via a workpiece carrier, which is propelled on integrated conveyor belts. The product to be assembled is linked by a RFID Tag attached to the work piece carrier and its RFID UID with a virtual assembly memory.

To control the modules of the assembly line, an agent is implemented in each assembly station. For example, in the third station the agent connects four different modules with one another, as illustrated in Fig. 3. The conveyor module (ATC) has an integrated RFID reader notifying the agent, via Modbus, when the workpiece carrier has entered the station. The agent then requests a receipt for the next assembly operation from the database. Once the agent receives the requested information, it processes and decides the appropriate assembly module that needs to be triggered. If a new part needs to be attached to the product, the agent will send the part name via OPC-UA to the pick by light feeding module system, located in the third station. At the same time, the agent will also send the operation information for the task along with its parameters to the assistance module via a TCP/IP interface as a string. These instructions will then be displayed on a screen for the worker to see. In the case that some bolts have already been attached to the product, which need to be tightened in the next process step, the agent will send a string to the cordless nutrunner. This nutrunner acts as a process module with an integrated control system. Depending on the information (drive type, torque, position) the nutrunner selects the correct tightening program. Each module notifies the agent when the assembly operation is completed, so that the agent can update the assembly memory. Once all possible assembly operations in the third station have been carried out, the 
agent signals the ATC to release the workpiece carrier and send the product to the next station.

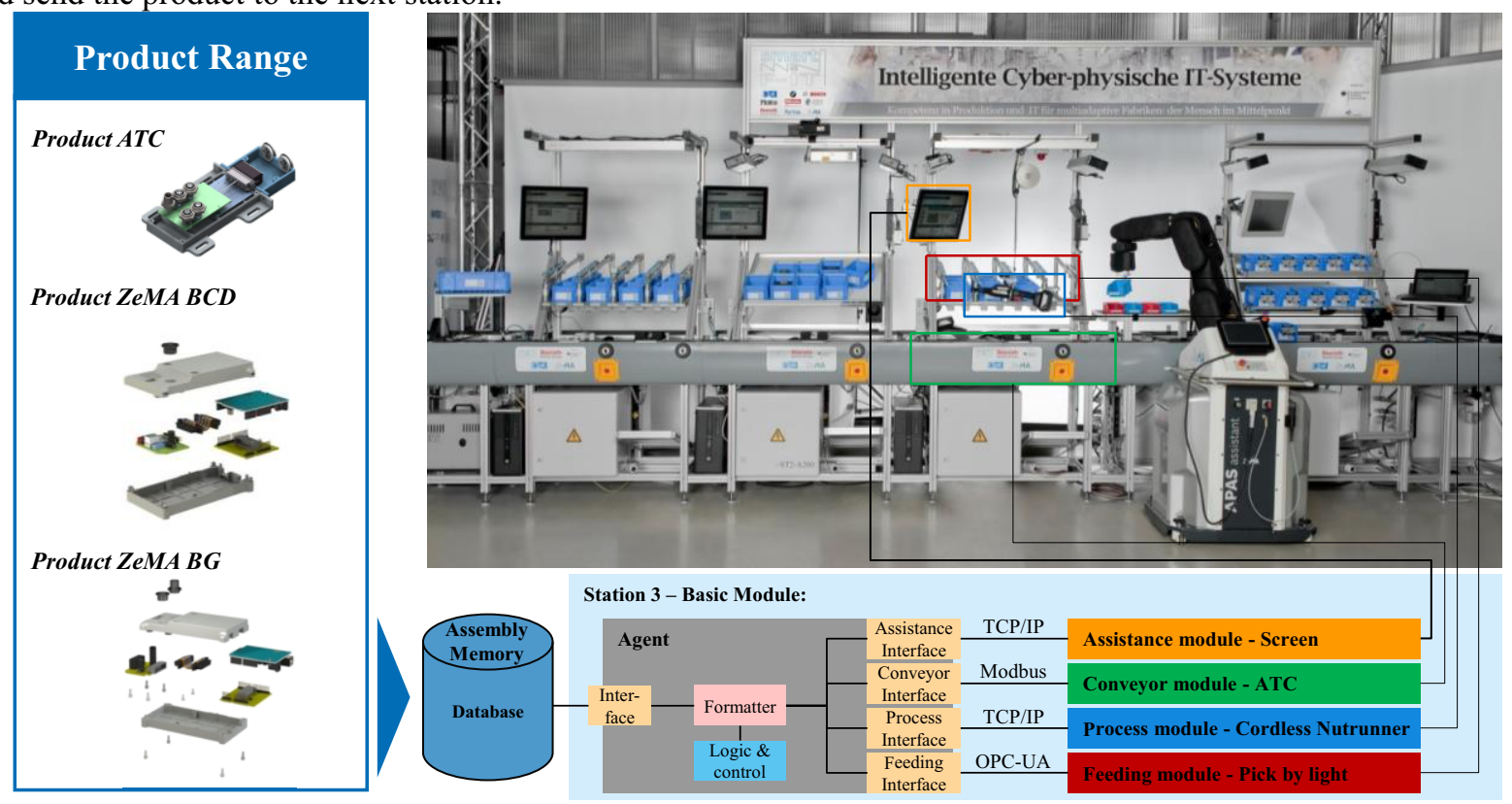

Figure 3: Implementation of the agent-based control architecture

\section{Summary and Outlook}

In this paper, an approach for a continuous information usage from planning to operation and control of a cyberphysical assembly system is presented. Due to the semantically coherent description of the assembly operations and a flexible control based on agent-systems, the assembly system is adaptable to upcoming changes from the product side. Furthermore, different protocol interfaces and the configuration of the agent enable the integration of new assembly modules. The agent works decentralized in each station as an information handler between the modules. On arrival, the product requests services from the station that are triggered by the agent based on the RFID UID and information stored in the assembly memory.

However, the information transfer from the virtual planning environment to the assembly memory database is not fully automated. Furthermore, information about the actual assembly system configuration, like production times, has to be returned to the virtual planning environment to keep it up-to-date. Therefore, a handler for the exchange between different software tools has to be developed.

\section{Acknowledgements}

This paper was written in the framework of the research project SmartF-IT that is funded by the German Federal Ministry of Education and Research (BMBF) and it is supervised by the lead partner German Aerospace Center (DLR) under the funding code 01S13015.

\section{References}

1. Y. Koren: The Global Manufacturing Revolution: Product-Process-Business Integration and Reconfigurable Systems. Wiley Series in Systems Engineering and Management (2010)

2. R. Müller, M. Vette, L. Hörauf, C. Speicher: Mechatronische Module als Befähiger für einendurchgängigen Informationsfluss bei der Planung und dem Betrieb von Cyber-Physischen Montagesystemen (VDI Mechatronik Conference, Germany 2015)

3. T. Bauernhansl: ARENA 2036 - The fourth industrial revolution in the automotive industry (SISAET 2015)

4. Lee, E. A.; Cyber Physical Systems: Design Challenges (Technical Report No. UCB/EECS-2008)

5. Smale, D.; Ratchev, S.: Application of a Reconfiguration Methodology for Multiple Assembly System Reconfigurations (IFIP Advances in Information and Communication Technology, 2010)

6. Sepehri, M.: Agent based approach for intelligent distribution control systems (2014)

7. Monostori, L.; Váncza, J.; Kumara, S.R.T.: AgentBased Systems for Manufacturing (Annals of the CIRP 2006)

8. Upadhyay, D. B.: A review paper on Multi agent base intelligent manufacturing system (IJAERS, 2014)

9. N. Thomopoulos, Assembly Line Planning and Control. Springer (2014)

10. [10]R. Müller, M. Vette, C. Speicher, L. Hörauf: Methodology for planning and operating cyberphysical assembly systems (FAIM Conference, England, 2015) 